\title{
Accelerometer-Based Navigation in Total Knee Arthroplasty for the Management of Extra-Articular Deformity and Retained Femoral Hardware: Analysis of Component Alignment
}

\author{
Andrea Cozzi Lepri ${ }^{1}$ Matteo Innocenti ${ }^{1}$ Fabrizio Matassi ${ }^{1}$ Marco Villano ${ }^{1}$ Roberto Civinini ${ }^{1}$ \\ Massimo Innocenti ${ }^{1}$ \\ ${ }^{1}$ Orthopaedic Clinic, University of Florence, NeuroMuscoloSkeletal \\ and Sense Organs Department, Orthopaedic Traumatologic Center, \\ Azienda Ospedaliero-Universitaria Careggi, Florence, Italy

\begin{abstract}
Address for correspondence Andrea Cozzi Lepri, MD, Orthopaedic Clinic, NeuroMuscoloSkeletal and Sense Organs Department, Ospedaliero-Universitaria Careggi, Largo P. Palagi 1, Florence 50139, Italy (e-mail: andreaco84a@gmail.com).
\end{abstract} \\ Orthopaedic Traumatologic Center, University of Florence, Azienda \\ Joints 2019;7:1-7.
}

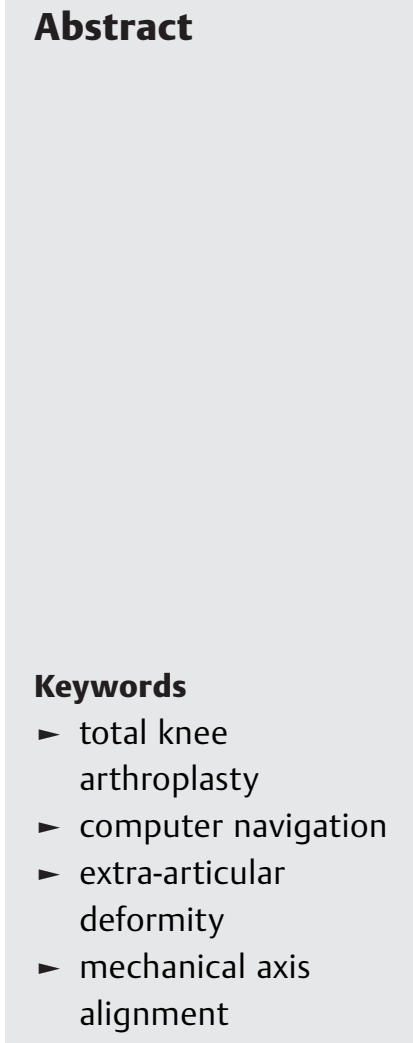

Purpose Recent advances in total knee arthroplasty (TKA) include an accelerometer portable system designed to improve component position and alignment. The purpose of this study is to evaluate whether accelerometer navigation system can be a valuable option in complex TKAs for extra-articular deformity of the lower limb or in case of retained femoral hardware.

Methods A group of 13 patients underwent TKA with an accelerometer navigation system. Three patients had a tibial extra-articular deformity, six had a femoral extraarticular deformity, and four had an intramedullary nail in the femur. Preoperative and postoperative mechanical axes were measured from full-length lower extremity radiographs to evaluate alignment. The alignment of prosthetic components in the frontal and sagittal planes was determined by postoperative radiographs.

Results At 30-days postoperative radiographic check, the hip knee ankle angle was within $2.0^{\circ}(0 \pm 1)$ of the neutral mechanical axis. The alignment of the tibial component on the frontal plane was $90.0^{\circ}$ (range 89-91) and on the sagittal plane $5.0^{\circ}$ (range 3-7). The alignment of the femoral component on the frontal plane was $90.0^{\circ}$ (range 89-91) and on the sagittal plane $3.0^{\circ}$ (range $0-5$ ).

Conclusion The alignment of the prosthetic components has been accurate and comparable to other navigation systems in literature without any increase in surgical times. The accelerometer-based navigation system is therefore a useful technique that can be used to optimize TKA alignment in patients with extra-articular deformity or with lower limb hardware, where the intramedullary guides cannot be applied.

Level of Evidence This is an observational study without a control group, Level III. received

July 27,2018

accepted

August 7, 2019

published online

October 11, 2019
DOI https://doi.org/

10.1055/s-0039-1697610.

ISSN 2282-4324.
Copyright (c) 2019 Georg Thieme Verlag License terms

KG Stuttgart · New York 


\section{Introduction}

Total knee arthroplasty (TKA) is a satisfactory consolidated procedure to improve function and to obtain a good relief from pain in osteoarthritis. The success and longevity of this procedure however is strictly correlated with lower limb alignment, components position, and ligament balancing. ${ }^{1-7}$

For decades, a stable knee with a neutrally aligned lower limb has been one of the primary goals of TKA because it was supposed to be important for successful clinical outcomes and implant survivorship. ${ }^{8}$ Therefore, the aim of mechanical alignment technique for TKA is not to restore the constitutional patient-specific alignment, ${ }^{9}$ but rather to systematically create a "biomechanically friendly prosthetic knee."

When osteoarthritis is combined with a femoral or tibial extra-articular deformity, performing a TKA and restoring neutral mechanical axis can turn the surgery into a challenge.

Femoral or tibial deformity for previous trauma or surgery may compromise the use of conventional intramedullary instrumentation for TKA because of distortion of the bony canal or the presence of hardware.

In these cases, many systems can support the surgeon to make the surgery accurate bypassing the problems related to deformity such as navigation, robotics, patients-specific instrumentation, or the use of extramedullary guide. However, they have some disadvantages related to the cost, to the complexity of their use, the learning curve, and the necessity of preoperative magnetic resonance or computed tomography.

Recent advances in TKA include an accelerometer portable system (iAssist, ZIMMER BIOMET, Warsaw, Indiana, United States) designed to improve component position and alignment, which is smart, easy to use, and cheap.

IAssist is an intelligent cutting block system that provides intraoperative matching of bone cuts and overall alignment at each surgical step, using an internal position-sensing technology integrated into microelectronic pods that attach to cutting blocks. This technology allows positioning the cutting blocks without intrusion into the intramedullary canal.

The purpose of this study is to evaluate whether iAssist system can be a valuable option in complex TKAs for extraarticular deformity of the lower limb or in case of retained femoral hardware.

Our hypothesis is that this new technology enables the surgeon to make accurate bone resections and restore the neutral mechanical axis in these complex TKAs where conventional intramedullary instrumentation could not be used.

\section{Methods}

\section{Patients}

This was a prospective study. At the Orthopaedic Clinic, University of Florence, a group of consecutive 13 patients with primary knee osteoarthritis associated with extraarticular deformity were recruited between May 2016 and September 2017 to undergo TKA with the iAssist navigation system. The mean inclusion criterion was a moderate to severe osteoarthritis associated to extra-articular deformity. For the knee to be included in the study, the extra-articular deformity had to be an angular deformity that should be corrected with a TKA without any violation of the collateral ligament insertion according to the Wang's criteria. ${ }^{10}$

Exclusion criteria included active infection, severe coincident hip arthritis, neurological disorders, prior knee arthroplasty or patellectomy, fixed deformity greater than $15^{\circ}$, needing of a previous osteotomy cause not meeting of the Wang's criteria, ${ }^{10}$ worker's compensation claims, cancer, metabolic bone disease, osteoporosis/osteopenia (diagnosed or treated with medication), and active immunosuppressive disorder requiring cytotoxic drugs, corticosteroids, or irradiation.

For each patient that was indicated for TKA, the abovelisted criteria were applied and those who met the inclusion/exclusion criteria were offered enrolment in the study.

The lead surgeon informed patients about the study objectives and the unique features of the iAssist surgical guidance system. Written consent for study participation was obtained from all the patients.

Our study series consisted of eight male and five female, with an average age of $63.7 \pm 2.3$. The mean body mass index was $28.6 \pm 1.3$. All selected patients had a Grade IV osteoarthritis according to Kellgren-Lawrence classification. Among these, according to Ahlbäck classification, six had a corresponding Grade IV, four had Grade V, and the remaining three patients had a Grade III osteoarthritis. Three patients had a tibial extra-articular deformity, six had a femoral extra-articular deformity, and four had intramedullary nail in the femur (-Fig. 1).

\section{Surgical Technique}

Our objective was to restore a neutral mechanical axis of the lower limb.

We also aim to obtain an alignment of the tibial component on the frontal plane within $90^{\circ}$ with respect to the tibial mechanical axis and on the sagittal plane within $5^{\circ}$ of tibial slope. For the femoral alignment, we aim to position the component on the frontal plane within $90^{\circ}$ with respect to the femoral mechanical axis and on the sagittal plane within $3^{\circ}$ of the femoral flexion.

Thirty minutes preoperative and 30 minutes postoperative a tranexamic acid intravenous (IV.) infusion $(1 \mathrm{mg} / \mathrm{kg}$ ) was administrated. Vancomycin $1 \mathrm{~g}$ IV was administrated within 2 hours before the surgery. Cefazolin $2 \mathrm{~g}$ IV was administrated 10 minutes before inflating the tourniquet. The lead surgeon (F.M.) was the same for all the patients. A tourniquet was applied at the thigh root and the tourniquet time was measured. A standard TKA was performed through a longitudinal anterior skin incision and a medial parapatellar arthrotomy.

IAssist does not require pins for optical tracking, as such, no additional incisions were made. Cutting blocks were affixed to the bone using the same pins as with conventional TKA. For the femur, a little spike was impacted for 2.5 to $3.5 \mathrm{~cm}$ at the mechanical axis entry point in the distal femur. The femoral reference pod was attached to the spike and the leg was then moved through a "stop-and-go" star configuration to acquire the hip center and orientation of the 

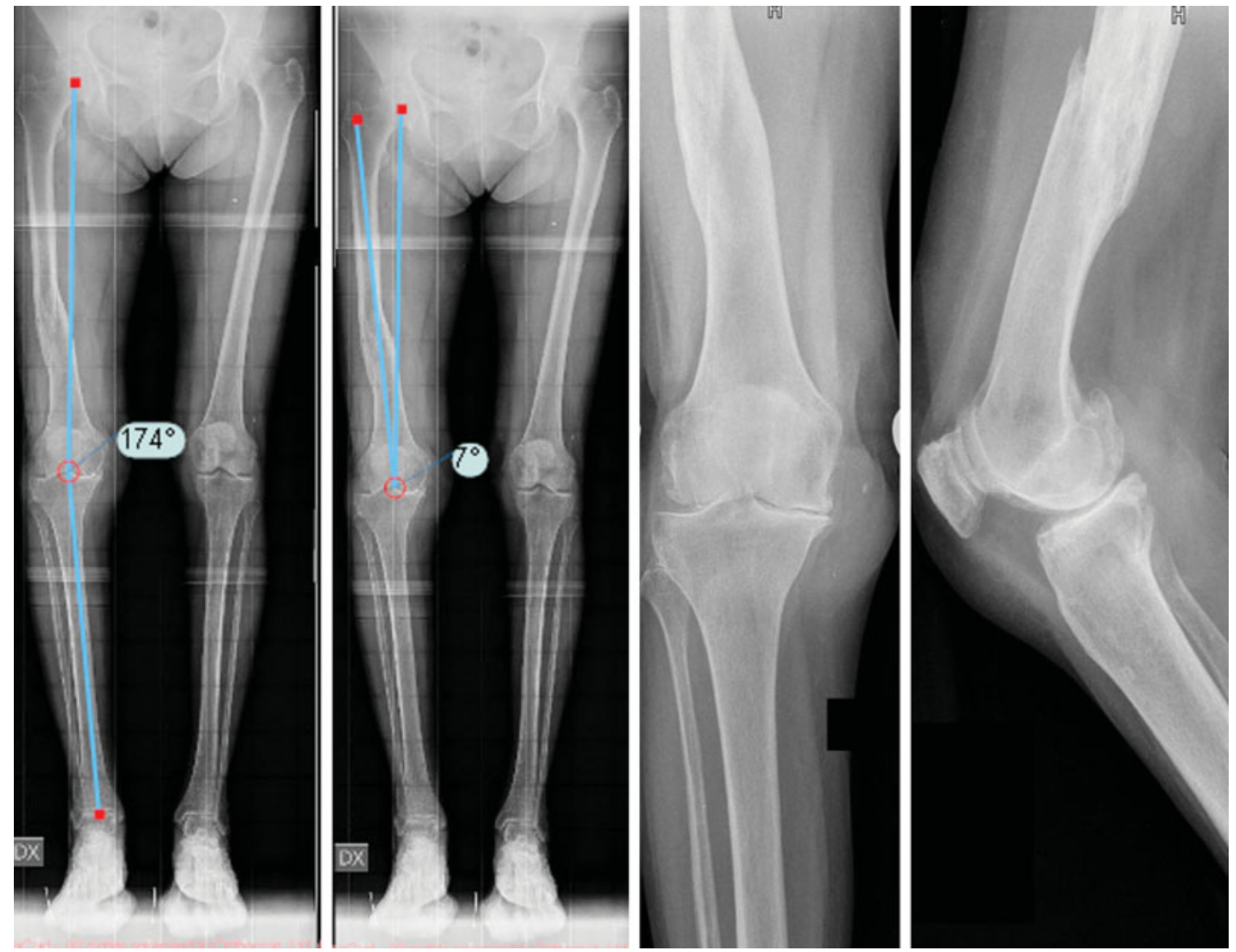

Fig. 1 Example of extra-articular deformity: mid-third triplanar femur shaft deformity.

mechanical axis. A femoral adjustment pod and cutting guide were then attached to the spike and the cut orientation (flexion/extension and varus/valgus) was modified with a distal femur cut perpendicular to the mechanical axis planned for all patients (-Fig. 2A). The distal femoral resection guide was then affixed to the distal femur and the spike and sleeve assembly were removed. The cut was made and a validation pod was used to confirm cut accuracy.

A similar process was used for the tibia. The proximal spike of the extramedullary guide was impacted at the mechanical axis entry point between the tibial spines. The distal portion of the guide consisted of self-centering clamps over the malleoli such that the instrumentation was fixed over the center of the ankle. The guide was then rotationally-oriented to be in line with the medial third of the tibial tubercle and was fixed in place. The leg was then brought into abduction, adduction, and back to neutral to allow the digitizer to register the tibial mechanical axis and transfer it to the pods attached to the tibial resection guide. The tibial resection guide was then adjusted to correct the coronal alignment, and the slope and depth of the cut were determined using a classic stylus (-Fig. 2B). Following resection, a validation tool was then used to confirm the cut orientation.
If necessary, an additional cut is performed to adjust the alignment according to the optimal position as recorded from the iAssist screen.

At the end of the procedure, with trial component in place, a ligament release was performed to balance the knee in extension and in flexion. In 11 cases, a posterior stabilized tibial insert was used and in two cases a constrained condylar knee was necessary to increase the stability of the knee because of larger flexion gap.

At the day of surgery, when anesthesia wore off, patients remained standing and started walking with two crutches with physiotherapist's assistance. First day postoperatively, patients started with continuous passive mobilization with ROM from $0^{\circ}$ to $90^{\circ}$ as tolerated. Between the third and fourth day postoperatively, patients were trained to walk upstairs and in the fourth to fifth day after surgery, they were dismissed.

\section{Outcome Measurements}

A clinical evaluation was conducted using the Knee Society score, recorded before surgery and in the postoperative period, after 1 month and 6 months.

Statistical analysis was performed using SPSS statistics software (IBM, Armonk, New York, United States). The 

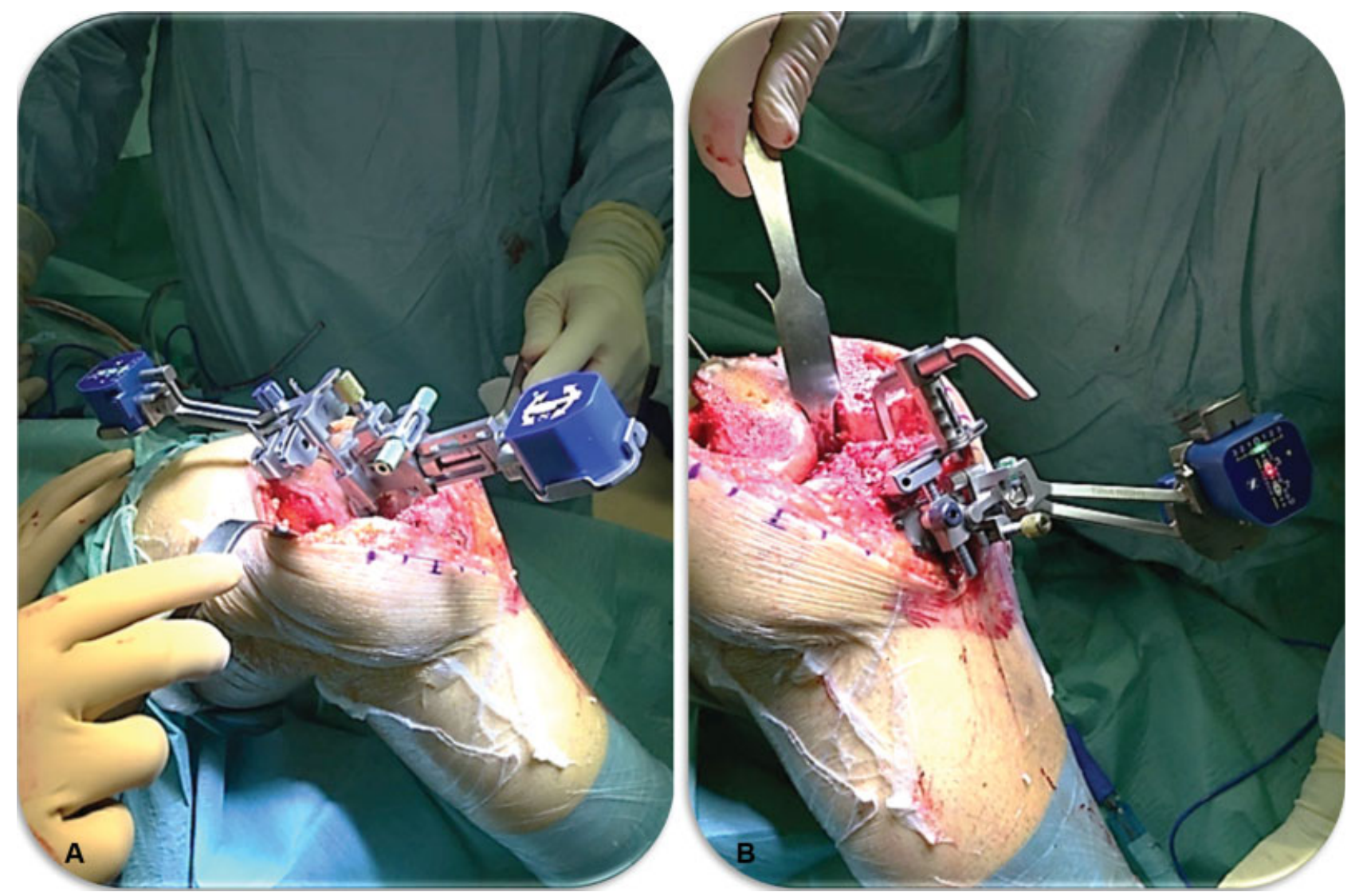

Fig. 2 iAssist system: femur validation and cut pods (A); tibia validation and cut pods (B).

clinical parameters were evaluated with the calculation of the mean and standard deviations (SD). Changes in the knee and function scores between preoperative and 1-month follow up and between preoperative and 6-months follow up were compared using the Student $t$-test, taking $p$-values of less than 0.05 as statistically significant with a $95 \%$ confidence interval.

Preoperatively and 30-days postoperatively, a radiological analysis was performed with full-length lower extremity radiographs in the frontal plane; in the sagittal plane a fulllength femur and tibia radiographs were performed too.

Additional projections in anterior-posterior and lateralside of the knee under load and an axial of the patella were also performed.

For each case, a preoperative planning has been set up by evaluating the mechanical and anatomical axes, measured with appropriate software very accurately (to within $1^{\circ}$ ), ${ }^{11}$ from full-length lower extremity radiographs (Maquet view) to evaluate alignment.

The center of the femoral head was defined using Moses circles. The preoperative center of the knee was determined using the midpoint of the two methods. The center of the line from the medial epicondyle border to the lateral epicondyle border at the level of the top of the intercondylar notch was demarcated. A second point at the center of the tibial plateau (measured at its widest level) was also demarcated. The midpoint of these two points in the center of the knee joint was determined to be the anatomical center of the knee. The anatomical center of the ankle was defined as the midpoint of the superior facet of the talus.

Five angles were measured. In the frontal plane the hip knee ankle (HKA) angle was measured to determine the mechanical axis of the lower limb as a difference from the neutral mechanical axis defined as $0^{\circ}$. The mechanic lateral distal femur angle and the medial proximal tibial angle were measured to determine the alignment of the femur and the tibia respectively in the frontal plane.

To measure the alignment in the sagittal plane we used the posterior distal femur angle and the posterior proximal tibial angle.

To determine the amount of bone to be resected from the femur, a line perpendicular to the mechanical axes of the femur was drawn through the femoral condyle on the preoperative full-length weight-bearing radiograph of the affected limb. If the anticipated femoral condylar resection violated the integrity of the insertion of either the medial or the lateral collateral ligament the patient was excluded from the study and a two-step surgery was performed with a corrective extra-articular osteotomy, followed by a TKA later. For the tibia, the same Wang's criteria was applied. $^{10}$

The five angles used in the preoperative radiographs were measured postoperatively to analyze the alignment of prosthetic components in the frontal and sagittal planes and were compared with intraoperative data provided by the navigation system (-Fig. $\mathbf{3}$ ). 

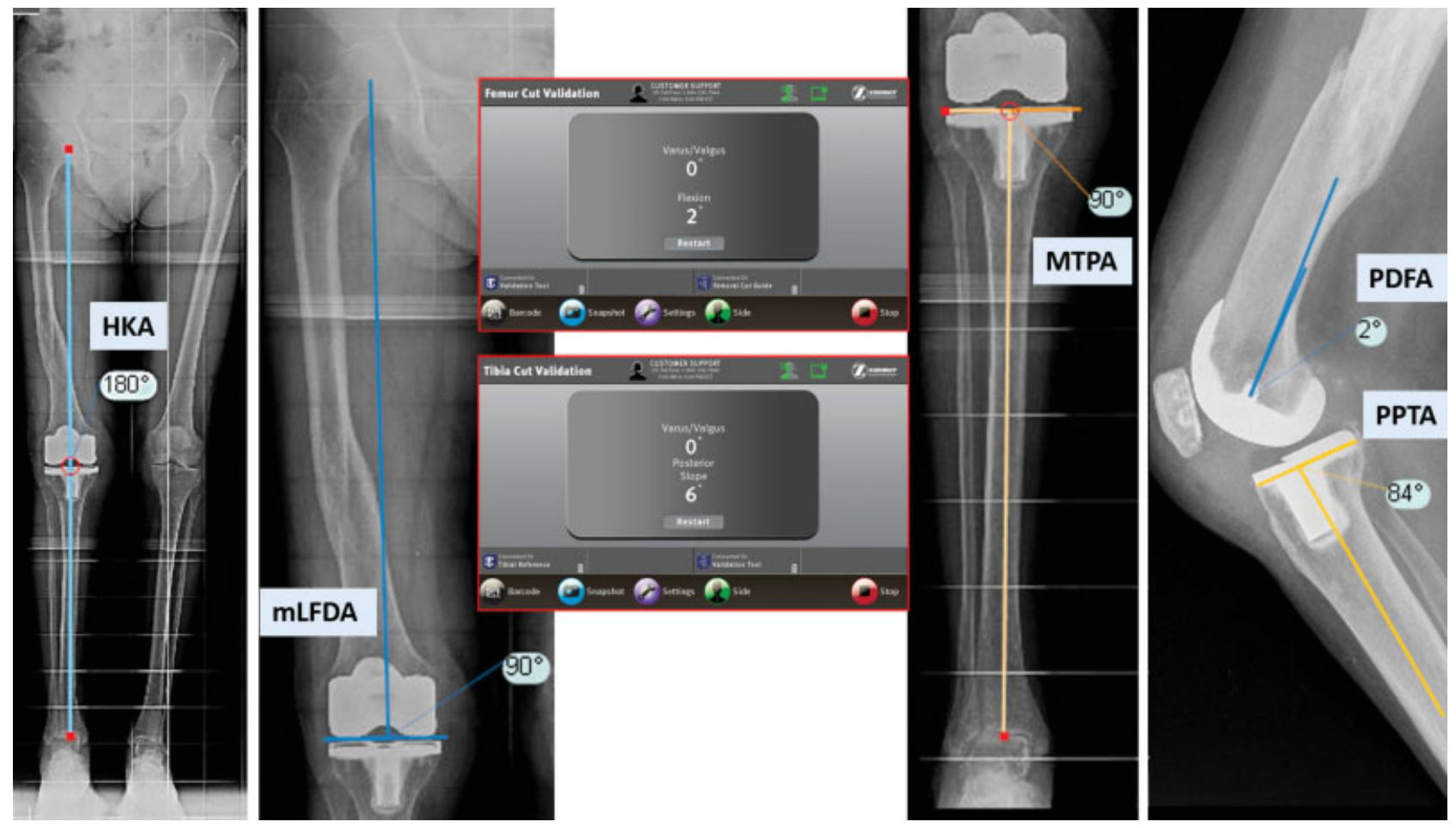

Fig. 3 Example of measurement of the five angles postoperatively and data provided by the navigation system intraoperatively for the same patient.

Table 1 Knee Society score and Functional score

\begin{tabular}{|l|l|l|l|l|l|}
\hline & Mean preoperative (SD) & Mean 1 month (SD) & $p$-Value & Mean 6 months (SD) & $p$-Value \\
\hline Knee score & $43.3( \pm 12.3)$ & $61.3( \pm 16.5)$ & $<0.08$ & $81.2( \pm 15.3)$ & $<0.05$ \\
\hline Function score & $36.7( \pm 12.5)$ & $58.8( \pm 16.2)$ & $<0.08$ & $79.3( \pm 16.5)$ & $<0.05$ \\
\hline
\end{tabular}

\section{Results}

Mean follow up was 5.7 months (1-6). No intraoperative and postoperative surgical complications were reported in the series. The mean tourniquet time was 62 minutes (range 5564 minutes). No blood transfusion has been necessary in the intra- and postoperative phase.

At the final follow up Knee and Functional scores increased from 43.3 to $81.2(p<0.05)$ and from 36.7 to 79.3 $(p<0.05)$, respectively ( - Table $\mathbf{1})$.

Lower extremity mechanical axis measurements from preoperative full-length weight-bearing radiographs revealed a range from $17^{\circ}$ varus to $15^{\circ}$ valgus.

At 30-days postoperative radiographic check, the HKA angle was within $2.0^{\circ}(0 \pm 1)$ of the neutral mechanical axis.

The alignment of the tibial component on the frontal plane was $90.0^{\circ}$ (range 89-91) and on the sagittal plane, $5.0^{\circ}$ (range 3-7). The alignment of the femoral component on the frontal plane was $90.0^{\circ}$ (range 89-91) and on the sagittal plane $3.0^{\circ}$ (range $0-5$ ) (-Table 2 ).

\section{Discussion}

With the iAssist system, we aimed to restore the neutral mechanical axis and to obtain an alignment of the tibial and femoral component on the frontal plane within $90^{\circ}$ with respect to the tibial and femoral mechanical axis and on the sagittal plane within $5^{\circ}$ of the tibial slope and within $3^{\circ}$ of the femoral flexion.

Our results showed that iAssist system enables the surgeon to restore the neutral mechanical axis components within $2^{\circ}$ of tolerance.

The tibial component was aligned on the frontal plane at an average of $90.0^{\circ}$ (range 89-91) and on the sagittal plane at $5.0^{\circ}$ (range 3-7). The alignment of the femoral component on

Table 2 Data provided from measurement in full-length lower extremity radiographs, postoperatively

\begin{tabular}{|l|l|}
\hline & $\begin{array}{l}\text { Data provided from } \\
\text { measurement in full-length } \\
\text { lower extremity radiographs }\end{array}$ \\
\hline $\begin{array}{l}\text { Postoperative mechanical } \\
\text { axis (HKA) }\end{array}$ & $0 \pm 1$ degree \\
\hline Postoperative mLFDA & 90.0 degree (range $89-91$ ) \\
\hline Postoperative MPTA & 90.0 degree (range $89-91$ ) \\
\hline Postoperative PDFA & 3.0 degree (range 0 -5) \\
\hline Postoperative PPTA & 5.0 degree (range $3-7$ ) \\
\hline
\end{tabular}

Abbreviations: HKA, hip knee ankle; mLFDA, mechanical lateral femoral distal angle; MPTA, medial proximal tibial angle; PDFA, posterior distal femur angle; PPTA, posterior proximal tibial angle. 
the frontal plane was $90.0^{\circ}$ (range 89-91) and on the sagittal plane, $3.0^{\circ}$ (range $0-5$ ).

Extra-articular deformity around the knee is a surgical challenge to fight with conventional instrumentation when performing a TKA.

In most of the cases, an extramedullary guide is used for tibial cuts. However, in case of deformity, having precise and secure anatomical landmarks to place the cutting block with the correct slope and alignment is difficult also with an extramedullary guide. On the other hand, an intramedullary guide is often used for the femur. Obviously, in case of retained hardware in the canal or in case of a metaphyseal-diaphyseal deformity, the rod is impossible to use. Furthermore, standard valgus alignment guide has limited angles of choice. Staged surgical procedures, including osteotomy of the femur or tibia, followed by traditional TKA, can be used to recreate the correct mechanical axis, nevertheless increasing the risk of complications. ${ }^{12}$ Therefore, in all these cases, having a system that bypasses the deformity could be helpful.

Computer-assisted stereotaxic navigation (CASN) was introduced as an adjunct to TKA surgery with the potential to improve positioning and alignment of the TKA prosthesis. Although recent meta-analyses demonstrated that the average coronal plane alignment after computer-assisted navigation TKA was not different from conventional TKA, the variability in the outcome was reduced. ${ }^{13-15}$ These metaanalyses concluded that it might be employed as a method of reducing error in prosthesis positioning, improving accuracy of postoperative lower-limb alignment.

Kuo et al ${ }^{16}$ and Klein et al ${ }^{17}$ reported an alignment within 1 to $2^{\circ}$ of femoral and tibial mechanical-articular angles in patients with extra-articular deformity or retained hardware underwent TKA using CASN.

Liu et $\mathrm{al}^{18}$ reported that CASN TKA for osteoarthritis with extra-articular deformity resulted in satisfactory outcomes. Still, despite of these encouraging data, CASN have some disadvantages. Use of trackers with transosseous pins enhances the risk of weakening the anterior cortex of femur and tibia ${ }^{19-21}$ and of additional bleeding; furthermore, the system requires a recording chamber of the signal and the surgeon needs to observe a monitor during the whole operation. Several studies underline major surgical times and a learning curve of approximately 30 cases to overcome the delay in operating time. ${ }^{22,23}$

Also CASN, the use of Robot technology shares with it the same disadvantages. ${ }^{24-26}$

Doubtless, the accuracy in alignment, the safety and the possibility of a tissue-sparing surgery make the Robot a valid tool in operating room. ${ }^{24,25} \mathrm{Kim}$ et al investigated the role of Robot-assisted surgery in intra-articular deformity, such as hemophilic arthropathy, ${ }^{26}$ showing excellent accuracy of lower limb and component alignment. However, they concluded that "expensive cost, additional preparation time, and longer operation time with similar clinical results in hemophilic arthropathy should be concerned." Furthermore, planning a surgery with Robot requires computed tomography (CT) scan that is not safe and comfortable for the patients. To use CT scans the system needs dedicated softwares and a variable period to create the template, whereas not everywhere softwares using the Robots are available and optimized for TKA. Both the technologies are expensive and not all the health care centers can afford it.

Patient-specific instrumentation (PSI) is another option in complex TKA. ${ }^{27,28}$ Roh et al reported some increase in error rate on CT-based PSI compared with MRI. ${ }^{29}$ However, creating the custom made cutting blocks needs almost 3 to 6 weeks, and during surgery no feedbacks for alignment or rotation are available. Many studies in the literature are discordant on outcomes obtained by the use of this system. ${ }^{28,30}$ Chen et $\mathrm{al}^{31}$ reported that the clinical outcomes were comparable between the PSI-TKA and conventionalTKA groups. Several authors report a minor accuracy compared with CASN. Lionberger et $\mathrm{al}^{28}$ showed that accuracy within $\pm 3^{\circ}$ from neutral alignment was achieved in $90 \%$ of the CASN TKA, compared with $80 \%$ among the patientspecific instrumentation group. Similar findings were found compared with manual versus PSI with an accuracy of $\pm 3^{\circ} .{ }^{\circ}$ Using the same accuracy, Nunley et al found $32 \%$ of PSI compared with conventional $40 \%{ }^{33}$

Our experience with this brand new tool seems to be efficient and adequate to achieve correct alignment during TKA. With respect to other expensive and complex technologies, this system is surely cheaper and easy to be applied for the management of complex TKA. No significant increments of surgical time and blood loss have been recorded in this series. The learning curve may be considered approximately five cases.

Our data are in line with the recent literature that have compared the accuracy in restoring the neutral mechanical axes of this internal position-sensing technology and conventional instrumentations. ${ }^{34}$

On sagittal plane, we positioned the tibial component with 3 to $7^{\circ}$ of slope and the femur component with a flexion from 0 to $5^{\circ}$. Kim et $\mathrm{al}^{35}$ found that flexions of the femoral component of $>3^{\circ}$ or sagittal alignment of the tibial component of $<0^{\circ}$ or a tibial slope of $>7^{\circ}$ were risk factors for failure. However, their measurements on the sagittal plane referred to anatomical axes on the postoperative short lateral side radiographs of the knee, according to the Knee Society Radiographic Evaluation System. Our measurements, instead, are evaluated on the lateral side femur and tibia into full-length radiographs, referring to mechanical and nonanatomical axes.

Our study presents some limitations. Number of cases consisted only of 13 patients, but this limitation is accounted for the small incidence of osteoarthritis associated with extra-articular eligibility for TKA without a previous osteotomy step. The follow up was short and we reported the Knee score and Functional score without any comparison with the outcome of conventional TKA cases. However, our study wanted to be a radiographic analysis of the iAssist ability to restore neutral mechanical axes in case of extra-articular deformities.

In conclusion, we can affirm that the accelerometer-based navigation system is a useful technique that can be used to optimize TKA alignment in patients with extra-articular 
deformity or with lower limb hardware, where the intramedullary guides cannot be applied, with a good component positioning and restoration of the neutral mechanical axis.

\section{Conflict of Interest}

None declared.

\section{References}

1 Insall JN, Ranawat CS, Aglietti P, Shine J. A comparison of four models of total knee-replacement prosthesis. J Bone Joint Surg Am 1976;58A:754-765

2 Insall JN, Binazzi R, Soudry M, Mestriner LA. Total knee arthroplasty. Clin Orthop Relat Res 1985;(192):13-22

3 Ranawat CS, Boachie-Adjei O. Survivorship analysis and results of total condylar knee arthroplasty. Eight- to 11-year follow-up period. Clin Orthop Relat Res 1988;(226):6-13

4 Knutson K, Lewold S, Robertsson O, Lidgren L. The Swedish knee arthroplasty register. A nation-wide study of 30,003 knees 19761992. Acta Orthop Scand 1994;65(04):375-386

5 Buechel FF Sr. Long-term followup after mobile-bearing total knee replacement. Clin Orthop Relat Res 2002;(404):40-50

6 Callahan CM, Drake BG, Heck DA, Dittus RS. Patient outcomes following tricompartmental total knee replacement. A metaanalysis. JAMA 1994;271(17):1349-1357

7 Civinini R, Carulli C, Matassi F, Villano M, Innocenti M. Total knee arthroplasty after complex tibial plateau fractures. Chir Organi Mov 2009;93(03):143-147

8 Whiteside LA. Principles of ligament balancing and alignment in total knee arthroplasty. In: Parvizi J, Cashman J, Goyal N, Morrey MC, Restrepo C. The Knee: reconstruction, replacement and revision. Towson: Data Trace Publishing Company; 2013;51(01):51-23

9 Bellemans J, Colyn W, Vandenneucker H, Victor J. The Chitranjan Ranawat award: is neutral mechanical alignment normal for all patients? The concept of constitutional varus. Clin Orthop Relat Res 2012;470(01):45-53

10 Wang JW, Wang CJ. Total knee arthroplasty for arthritis of the knee with extra-articular deformity. J Bone Joint Surg Am 2002; 84(10):1769-1774

11 Graves SE, Davidson D, Ingerson L, et al. The Australian Orthopaedic Association National Joint Replacement Registry. Med J Aust 2004;180(5, Suppl):S31-S34

12 Papadopoulos EC, Parvizi J, Lai CH, Lewallen DG. Total knee arthroplasty following prior distal femoral fracture. Knee 2002; 9(04):267-274

13 Bauwens $\mathrm{K}$, Matthes $\mathrm{G}$, Wich $\mathrm{M}$, et al. Navigated total knee replacement. A meta-analysis. JBone Joint Surg Am 2007;89 (02):261-269

14 Hetaimish BM, Khan MM, Simunovic N, Al-Harbi HH, Bhandari M, Zalzal PK. Meta-analysis of navigation vs conventional total knee arthroplasty. J Arthroplasty 2012;27(06):1177-1182

15 Rebal BA, Babatunde OM, Lee JH, Geller JA, Patrick DA Jr, Macaulay W. Imageless computer navigation in total knee arthroplasty provides superior short term functional outcomes: a meta-analysis. J Arthroplasty 2014;29(05):938-944

16 Kuo CC, Bosque J, Meehan JP, Jamali AA. Computer-assisted navigation of total knee arthroplasty for osteoarthritis in a patient with severe posttraumatic femoral deformity. JArthroplasty 2011;26(06):976.e17-976.e20
17 Klein GR, Austin MS, Smith EB, Hozack WJ. Total knee arthroplasty using computer-assisted navigation in patients with deformities of the femur and tibia. J Arthroplasty 2006;21(02):284-288

18 Liu Z, Pan X, Zhang X. Total knee arthroplasty using navigation system for severe osteoarthritis with extra-articular deformity. Eur J Orthop Surg Traumatol 2013;23(01):93-96

19 Kamara E, Berliner ZP, Hepinstall MS, Cooper HJ. Pin site complications associated with computer-assisted navigation in hip and knee arthroplasty. J Arthroplasty 2017;32(09):2842-2846

20 Minoda Y, Watanabe K, Iwaki H, Takahashi S, Fukui M, Nakamura $\mathrm{H}$. Theoretical risk of anterior femoral cortex notching in total knee arthroplasty using a navigation system. J Arthroplasty 2013; 28(09):1533-1537

21 Minoda Y, Kobayashi A, Iwaki $\mathrm{H}$, et al. The risk of notching the anterior femoral cortex with the use of navigation systems in total knee arthroplasty. Knee Surg Sports Traumatol Arthrosc 2010;18 (06):718-722

22 Jenny JY, Miehlke RK, Giurea A. Learning curve in navigated total knee replacement. A multi-centre study comparing experienced and beginner centres. Knee 2008;15(02):80-84

23 Jenny JY, Picard F. Learning navigation-learning with navigation. A review. SICOT J 2017;3:39

24 Siebert W, Mai S, Kober R, Heeckt PF. Technique and first clinical results of robot-assisted total knee replacement. Knee 2002;9 (03):173-180

25 Bellemans J, Vandenneucker H, Vanlauwe J. Robot-assisted total knee arthroplasty. Clin Orthop Relat Res 2007;464(464): 111-116

26 Kim SM, Park YS, Ha CW, Lim SJ, Moon YW. Robot-assisted implantation improves the precision of component position in minimally invasive TKA. Orthopedics 2012;35(09):e1334-e1339

27 Mattei L, Pellegrino P, Calò M, Bistolfi A, Castoldi F. Patient specific instrumentation in total knee arthroplasty: a state of the art. Ann Transl Med 2016;4(07):126

28 Lionberger DR, Crocker CL, Chen V. Patient specific instrumentation. J Arthroplasty 2014;29(09):1699-1704

29 Roh YW, Kim TW, Lee S, Seong SC, Lee MC. Is TKA using patientspecific instruments comparable to conventional TKA? A randomized controlled study of one system. Clin Orthop Relat Res 2013;471(12):3988-3995

30 Chan WC, Pinder E, Loeffler M. Patient-specific instrumentation versus conventional instrumentation in total knee arthroplasty. J Orthop Surg (Hong Kong) 2016;24(02):175-178

31 Chen JY, Chin PL, Tay DK, Chia SL, Lo NN, Yeo SJ. Functional outcome and quality of life after patient-specific instrumentation in total knee arthroplasty. J Arthroplasty 2015;30(10):1724-1728

32 Lin WP, Lin J, Horng LC, Chang SM, Jiang CC. Quadriceps-sparing, minimal-incision total knee arthroplasty: a comparative study. J Arthroplasty 2009;24(07):1024-1032

33 Nunley RM, Ellison BS, Ruh EL, et al. Are patient-specific cutting blocks cost-effective for total knee arthroplasty? Clin Orthop Relat Res 2012;470(03):889-894

34 Kinney MC, Cidambi KR, Severns DL, Gonzales FB. Comparison of the iAssist handheld guidance system to conventional instruments for mechanical axis restoration in total knee arthroplasty. J Arthroplasty 2018;33(01):61-66

35 Kim YH, Park JW, Kim JS, Park SD. The relationship between the survival of total knee arthroplasty and postoperative coronal, sagittal and rotational alignment of knee prosthesis. Int Orthop 2014;38(02):379-385 